\title{
Thermodynamics and kinetics of the undercooled liquid and the glass transition of the $\mathrm{Zr}_{41.2} \mathrm{Ti}_{13.8} \mathrm{Cu}_{12.5} \mathrm{Ni}_{10.0} \mathrm{Be}_{22.5}$ alloy
}

\author{
R. Busch, ${ }^{\text {a) }}$ Y. J. Kim, and W. L. Johnson \\ W. M. Keck Laboratory of Engineering Materials, California Instilute of Technology, \\ Pasadena, California 91125
}

(Received 29 August 1994; accepted for publication 20 December 1994)

\begin{abstract}
Differential scanning calorimetry (DSC) was used to determine the thermodynamic functions of the undercooled liquid and the amorphous phase with respect to the crystalline state of the $\mathrm{Zr}_{41.2} \mathrm{Ti}_{13.8} \mathrm{Cu}_{12.5} \mathrm{Ni}_{10.0} \mathrm{Be}_{22.5}$ bulk metallic glass forming alloy. The specific heat capacities of this alloy in the undercooled liquid, the amorphous state and the crystal were determined. The differences in enthalpy, $\Delta H$, entropy, $\Delta S$, and Gibbs free energy, $\Delta G$, between crystal and the undercooled liquid were calculated using the measured specific heat capacity data as well as the heat of fusion. The results indicate that the Gibbs free energy difference between metastable undercooled liquid and crystalline solid, $\Delta G$, stays small compared to conventional metallic glass forming alloys even for large undercoolings. Furthermore, the Kauzmann temperature, $T_{K}$, where the entropy of the undercooled liquid equals to that of the crystal, was determined to be $560 \mathrm{~K}$. The Kauzmann temperature is compared with the experimentally observed rate-dependent glass transition temperature, $T_{g}$. Both onset and end temperatures of the glass transition depend linearly on the logarithm of the heating rate based on the DSC experiments. Those characteristic temperatures for the kinetically observed glass transition become equal close to the Kauzmann temperature in this alloy, which suggests an underlying thermodynamic glass transition as a lower bound for the kinetically observed freezing process. (C) 1995 American Institute of Physics.
\end{abstract}

\section{INTRODUCTION}

Recently, new families of multicomponent glass forming alloys such as La-Al-Ni, ${ }^{1}$ Zr-Ni-Al-Cu, ${ }^{2}$. and $\mathrm{Zr}-\mathrm{Ti}-\mathrm{Cu}-\mathrm{Ni}-\mathrm{Be}^{3}$ have been found which exhibit extraordinary glass forming ability. Cooling rates of less than $100 \mathrm{~K} / \mathrm{s}$ are usually sufficient to suppress nucleation of crystalline compounds and thus form a bulk metallic glass (BMG) in these alloy systems. For the particular $\mathrm{Zr}_{41.2} \mathrm{Ti}_{13.8} \mathrm{Cu}_{12.5} \mathrm{Ni}_{10.0} \mathrm{Be}_{22.5}$ alloy, it was even shown that the melt freezes to a glass applying cooling rates lower than 2 $\mathrm{K} / \mathrm{s}$ if heterogeneous nucleation is avoided by containerless processing. ${ }^{4}$ Sample sizes range up to $25 \mathrm{~mm}$ in the smallest dimension. This brings BMG close to technical applicability because of their unique features, such as high processability in the undercooled liquid regime and significantly increased yield strength below the glass transition. ${ }^{5}$

Due to the high thermal stability of undercooled liquids of $B M G$ formers, detailed studies of the thermophysical properties of metallic melts in the whole undercooled liquid region become possible. These thermophysical properties include specific heat capacity, viscosity, surface tension, and thermal expansion coefficient. Furthermore, in contrast to most conventional metallic glass formers, the glass transition can be investigated in a broad range of time scales.

In this article we focus on thermodynamics and the glass transition of the $\mathrm{Zr}_{41.2} \mathrm{Ti}_{13.8} \mathrm{Cu}_{12.5} \mathrm{Ni}_{10.0} \mathrm{Be}_{22.5}$ alloy, which is the best bulk glass former known so far. In order to determine the thermodynamic properties of the undercooled liquid and the amorphous phase in this alloy we used differential scanning calorimetry (DSC) to measure specific heat capaci-

a) Electronic mail: busch@hyperfine.caltech.edu ties and heats of transformation close to the glass transition temperature, $T_{g}$, and at the melting point, $T_{m}$. From the measurements, the thermodynamic functions are calculated as a function of temperature. This leads to the determination of the Kauzmann temperature ${ }^{6}$ which is shown to be the lower bound for the kinetically observed glass transition.

\section{EXPERIMENTAL METHODS}

Amorphous alloy ingots, with the nominal composition $\mathrm{Zr}_{41.2} \mathrm{Ti}_{13.8} \mathrm{Cu}_{12.5} \mathrm{Ni}_{10.0} \mathrm{Be}_{22.5}$, were prepared from a mixture of the elements of purity ranging from $99.5 \%$ to $99.9 \%$ by induction melting on a water cooled silver boat under a Tigettered argon atmosphere. Samples of $20-40 \mathrm{mg}$ were investigated in a Perkin-Elmer DSC7. Prior to every experiment, the DSC was evacuated to a pressure of $1 \mathrm{~Pa}$ and purged several times with $99.9999 \%$ Ar. Each sample was heated up above the glass transition using a rate of $0.33 \mathrm{~K} / \mathrm{s}$ and cooled with the rate of $3.3 \mathrm{~K} / \mathrm{s}$ back to room temperature to ensure the same thermal history for all samples. The calorimeter was recalibrated for each heating rate with indium and zinc standards. A second run for each specimen was directly carried out after the first cycle without changing the conditions of the measurement to construct a baseline. Measurements of absolute values of the specific heat capacity in the amorphous alloys up to $593 \mathrm{~K}$ and the crystallized samples up to $893 \mathrm{~K}$ were undertaken. To do this, the sample was heated with $0.33 \mathrm{~K} / \mathrm{s}$ to a certain temperature and held there for $180 \mathrm{~s}$. The resulting step in heat flux was compared with the signal of a sapphire standard and the empty $\mathrm{Cu}$ pan. This procedure was done every $20 \mathrm{~K}$. In addition, DSC mea- 


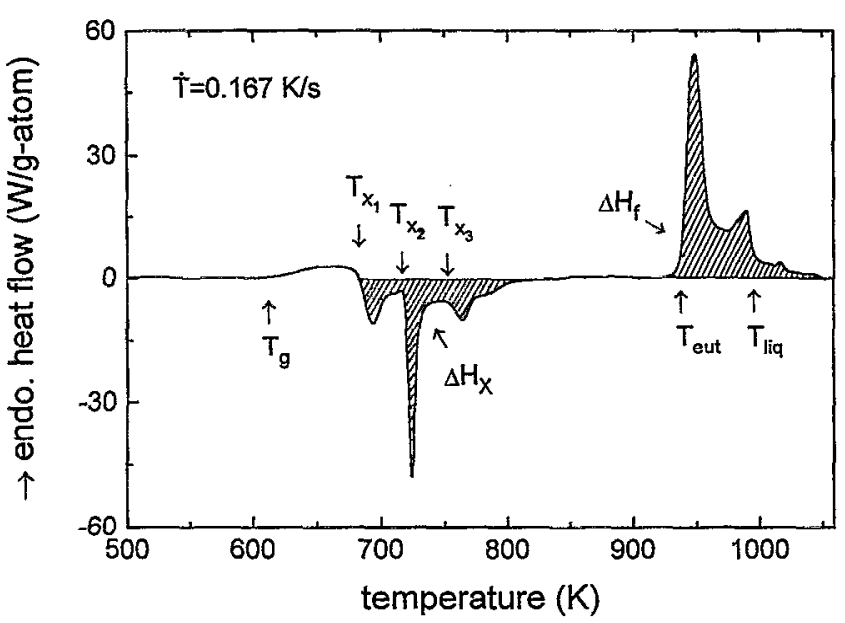

FIG. 1. DSC thermogram of the amorphous $\mathrm{Zr}_{41.2} \mathrm{Ti}_{13.8} \mathrm{Cu}_{12.5} \mathrm{Ni}_{10.0} \mathrm{Bc}_{22.5}$ samplc alloy at a heating rate of $0.167 \mathrm{~K} / \mathrm{s}$ indicating the crystallization with the heat release, $\Delta H_{x}$, and subsequent melting with the heat of fusion, $\Delta H_{f}$. Additionally marked are the onset of the glass transition, $l_{g}^{\text {onset }}$, the onsets of the three crystallization steps $T_{x_{i}}(i=1-3)$, the eutectic temperature, $T_{\text {eut }}$, and the liquidus temperature, $T_{\text {liq }}$.

surements were also carried out in a high-temperature calorimeter of type Seteram DSC $2000 \mathrm{~K}$ using $99.999 \% \mathrm{Ar}$. Runs of the empty crucibles taken prior to the experiments were subtracted from the measurements to obtain a straight baseline. Care was taken to remove the air prior to the runs.

\section{EXPERIMENTAL RESULTS}

The glass transition and crystallization behavior of the $\mathrm{Zr}_{41.2} \mathrm{Ti}_{13.8} \mathrm{Cu}_{12.5} \mathrm{Ni}_{10.0} \mathrm{Be}_{22.5}$ alloy were monitored in DSC scans for various heating rates. Figure 1 shows the DSC thermogram for a sample heated up with $0.167 \mathrm{~K} / \mathrm{s}$. It exhibits the endothermal heat effect due to the glass transition and three characteristic steps of heat release, indicating the successive slepwise transformations at $T_{x_{1}}, T_{x_{2}}$, and $T_{x_{3}}$ from the metastable. undercooled liquid state into the crystalline compounds at 680,720 , and $746 \mathrm{~K}$, respectively. Upon further heating, the crystallized sample finally starts to melt at the eutectic temperature $T_{\text {eut }}=937 \mathrm{~K}$, followed by complete melting at the liquidus temperature $T_{\mathrm{liq}}=993 \mathrm{~K}$.

The onset temperatures of the glass transition, $T_{g}$, and the transformation peaks, $T_{x(1,2,3)}$, appear to be strongly dependent on the heating rates. The heats of transformation determined by integrating the peak areas show a slight heating rate dependence as well. In Fig. 2 the specific heat capacity $\left(c_{p}\right)$ of the amorphous phase throughout the glass transition into the undercooled liquid is presented with respect to the crystalline solid for different heating rates. The absolute values for $c_{p}$ of the crystal and the amorphous alloy were measured with respect to sapphire standards. Below the onset temperature of the glass transition, the specific heat capacity of the amorphous phase does not change for different heating rates and it is only approximately $1 \mathrm{~J} / \mathrm{g}$-atom. $\mathrm{K}$ larger than the specific heat capacity of the crystal, as Fig. 2 indicates. Upon undergoing the glass transition, the track of the specific heat capacity becomes dependent on heating rate. This can

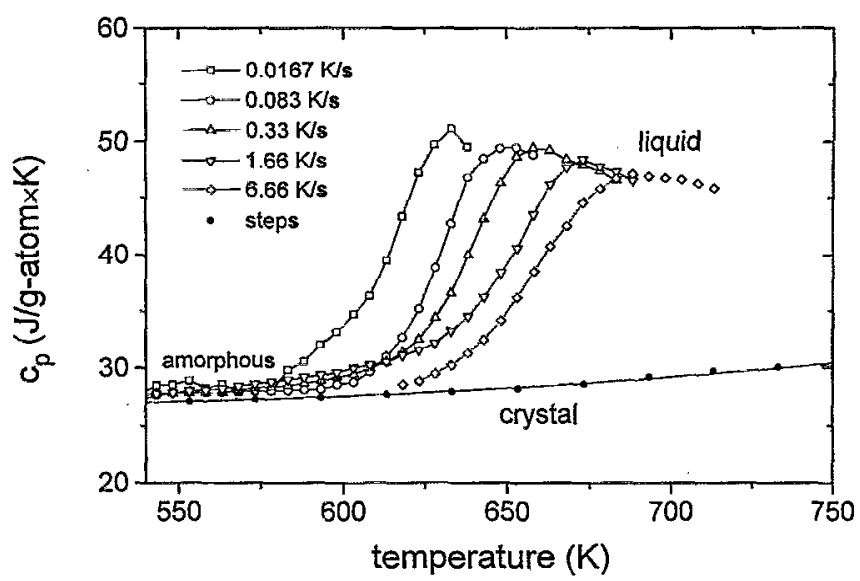

FIG. 2. Course of the specific heat capacity from the amorphous phase throughout the glass transition into the undercooled liquid for different heating rates. The snecific heat capacity data of the crystallized samples were measured in steps of $20 \mathrm{~K}$ in reference to a sapphire standard.

be attributed to the fact that relaxation times which are related to structural relaxations in the glass transition region are within the time of the experiment. The temperature at which the glass begins to respond to the temperature increase is defined by the onset of the glass transition $T_{g}^{\text {unsel }}$. The end temperature, $T_{g}^{\text {end }}$, above which the sample can fully equilibrate into the metastable undercooled liquid state during heating, is rate dependent as well. All the samples reach the undercooled liquid regime at different end temperatures. From there on they follow the same specific heat capacity curve. It can be clearly seen in Fig. 2 that $c_{p}$ in the undercooled liquid is a well defined decreasing function with increasing temperature. The temperature range in which the undercooled liquid can be observed in a DSC experiment is limited by the crystallization of the sample.

The crystallization of the sample is also rate dependent. This is caused by the fact that nucleation is a thermally activated process, whereas the rate dependence of the kinetic glass transition is due to the relaxation processes in the glass transition region.

With increasing heating rate the crystallization peaks are shifted to higher temperatures. Since the total heat release during crystallization measures the enthalpy difference between undercooled liquid and the crystalline state, we expect an increasing overall heat of crystallization with increasing heating rate. Table I summarizes the different characteristic temperatures and the measured enthalpies of transformation depending on heating rate as determined with the PerkinElmer DSC7. In particular, the heat release during the second transformation step is slightly dependent on the heating rate.

The heat of fusion is determined by the Seteram DSC $2000 \mathrm{~K}$ (see Fig. 1), at a rate of $0.167 \mathrm{~K} / \mathrm{s}$, and amounts to $8.2 \mathrm{~kJ} / \mathrm{g}$-atom that is raised in two steps of 5.4 and $2.8 \mathrm{~kJ} / \mathrm{g}$ atom at the eutectic and liquidus temperature, respectively. The total heat release during crystallization was $5.5 \mathrm{~kJ} / \mathrm{g}$ atom for this heating rate. 
TABLE L. Onset and end temperature $T_{g}^{\text {onset }}$ and $T_{s}^{\text {end }}$ of the glass transition depending on heating rate measured with the Perkin-Elmer DSC7. In addition, the onset temperatures and heat releases of the three crystallization steps depending on heating rate are listed.

\begin{tabular}{lccccc}
\hline \hline Temperatures and enthalpies & \multicolumn{5}{c}{ Heating rates $\dot{T}(\mathrm{~K} / \mathrm{s})$} \\
\hline & 0.0167 & 0.083 & 0.33 & 1.67 & 6.67 \\
$T_{g}^{\text {onset }}(\mathrm{K})$ & 602 & 614 & 620 & 631 & 636 \\
$T_{g}^{\text {end }}(\mathrm{K})$ & 628 & 643 & 653 & 668 & 680 \\
$T_{x_{1}}(\mathrm{~K})$ & 644 & 666 & 692 & 712 & 726 \\
$\Delta H_{x_{1}}(\mathrm{~kJ} / \mathrm{g}$-atom) & 1.1 & 1.2 & 1.2 & 1.2 & 1.2 \\
$T_{x_{2}}(\mathrm{~K})$ & 694 & 710 & 731 & 750 & 769 \\
$\Delta H_{x_{2}}(\mathrm{~kJ} / \mathrm{g}$-atom) & 1.2 & 2.6 & 3.0 & 3.4 & 3.6 \\
$T_{x_{3}}(\mathrm{~K})$ & 710 & 736 & 763 & 793 & 827 \\
$\Delta H_{x_{3}}(\mathrm{~kJ} / \mathrm{g}$-atom) & 2.8 & 1.4 & 1.4 & 1.3 & 1.4 \\
\hline \hline
\end{tabular}

\section{DISCUSSION}

\section{A. The thermodynamic functions of the undercooled liquid}

The thermodynamic functions of the $\mathrm{Zr}_{41.2} \mathrm{Ti}_{13.8} \mathrm{Cu}_{12.5} \mathrm{Ni}_{10.0} \mathrm{Be}_{22.5}$ alloy as a function of temperature are calculated based on the measured specific heat capacity data, which are shown in Fig. 3. The specific heat capacity of the amorphous phase is marked with triangles. The specific heat capacity data of the undercooled liquid above the glass transition (circles), obtained before crystallization upon heating the alloy, show about twice the value of the amorphous phase. They are a decreasing function with increasing temperature. The specific heat capacities that were obtained throughout the glass transition are not presented in Fig. 3, becauise they do not represent a thermodynamic equilibrium or metastable equilibrium state. The specific heat capacity above the eutectic temperature was measured with a modified Perkin-Elmer DSC7 by Fecht ${ }^{7}$ and does not exceed $41 \mathrm{~J} / \mathrm{g}$-atom $\cdot \mathrm{K}$ (diamond). Finally, the specific heat capacity of the crystallized alloy is also marked (squares).

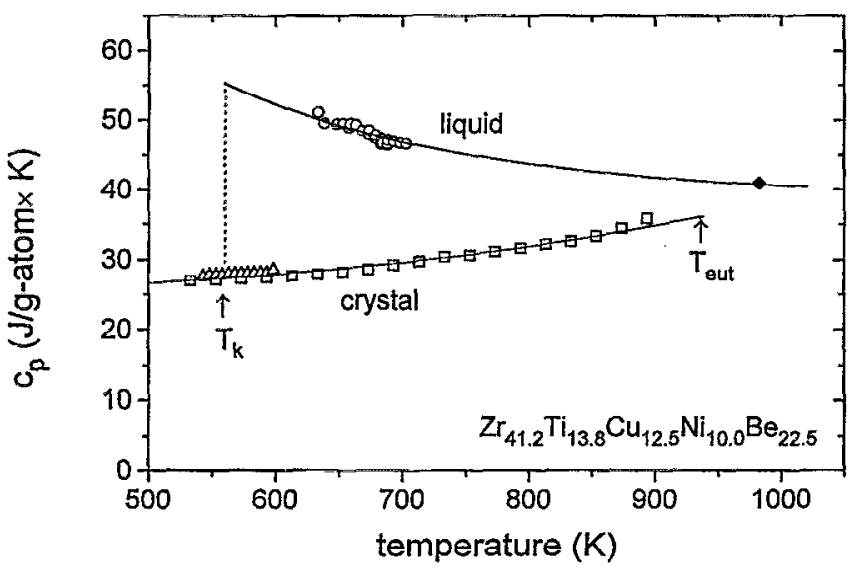

FIG. 3. Measured specific heat capacity of the undercooling liquid $(O)$ ), the amorphous phase $(\Delta)$, and the crystal $(\square)$. The specific heat capacity curves of the undercooled liquid and the crystal are fitted to the data.
According to Kubaschewski et al. ${ }^{8}$ the temperature dependence of the specific heat capacity of the undercooled liquid far above the Debye temperature can be expressed mainly as a $1 / T^{2}$ law as follows:

$$
c_{p}=3 R+b \cdot T+c \cdot T^{-2} \text {. }
$$

This has been successfully applied in various calculation-of-phase-diagram (CALPHAD) calculations to describe the temperature dependence of the specific heat capacity in the undercooled liquid. ${ }^{9,10}$ The fits to the specific heat capacity data for the crystal and the undercooled liquid are added in Fig. 3. The specific heat capacity of the undercooled liquid obeys the following equation:

$c_{p}^{\mathrm{liq}}=3 R+\left(\frac{7.5 \times 10^{-3} \cdot T}{K}+\frac{8.17 \times 10^{6}}{T^{2}} \cdot K^{2}\right) \frac{J}{\mathrm{~g} \text {-atom } \cdot \mathrm{K}}$.

The specitic heat capacity difference between the liquid and the crystal close to the melting point is less than $5 \mathrm{~J} / \mathrm{g}$ atom $\cdot \mathrm{K}$.

Since the temperature dependence of the specific heat capacity in the $\mathrm{Zr}_{41.2} \mathrm{Ti}_{13.8} \mathrm{Cu}_{12.5} \mathrm{Ni}_{10.0} \mathrm{Be}_{22.5}$ alloy is known for the noncrystalline and crystalline state as well, the Gibbs free energy of undercooled liquid with respect to the crystal, $\Delta G_{l-x}(T)$, can be calculated by integrating the specific heat capacity difference according to

$$
\begin{aligned}
\Delta G_{l-x}(T)= & +\Delta H_{f}-\Delta S_{f} \cdot T_{0}-\int_{T}^{T_{0}} \Delta c_{p}^{l-x}\left(T^{\prime}\right) d T^{\prime} \\
& +T \int_{T}^{T_{0}} \frac{\Delta c_{p}^{l-x}\left(T^{\prime}\right)}{T^{\prime}} d T^{\prime}
\end{aligned}
$$

in which $\Delta H_{f}$ and $\Delta S_{f}$ are the enthalpy and entropy of fusion, respectively, at the temperature $T_{0} . T_{0}$ is the temperature where the Gibbs free energy of the crystal is equal to the Gibbs free energy of the liquid. $\Delta c_{p}^{l-x}$ is the difference in specific heat capacity between liquid and solid. Even though $T_{0}$ is not exactly known for our alloy, from the pattern of the DSC track on melting we can assume that the alloy is quite far away from the eutectic composition. This means that $T_{0}$ is located between $T_{\text {eut }}$ and $T_{\text {liq }}$ and is likely to be very close 


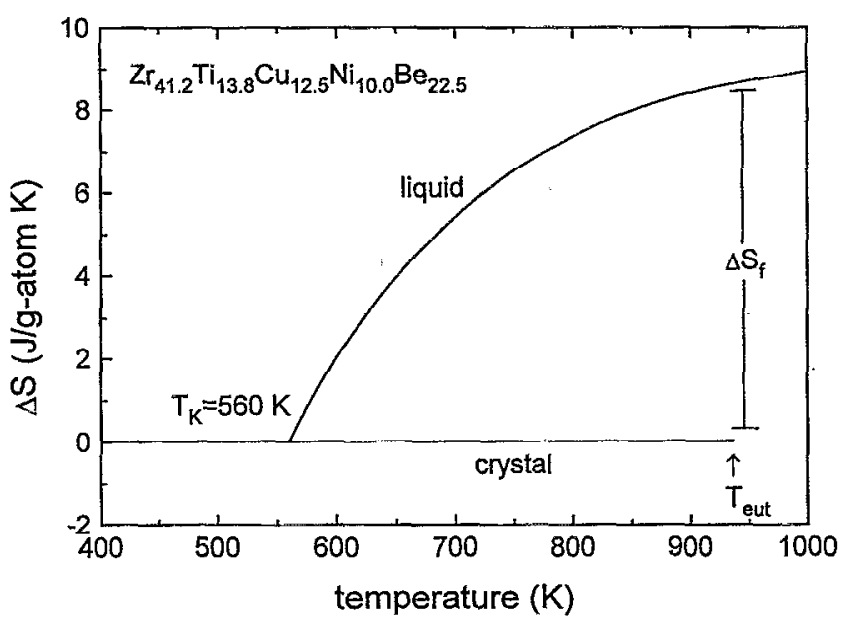

FIG. 4. Entropy of the undercooled liquid with respect to the crystal, including the cntropy of fusion, $\Delta S_{f}$, and the Kauzmann temperature, $T_{K}$.

to the eutectic temperature. In our calculations we use a value of $T_{0}=948 \mathrm{~K}$ that corresponds to the maximum of the first melting peak. For the determination of the total $\Delta S(T)$, $\Delta H(T)$, and $\Delta G(T)$, the error will be small, especially for large undercooling $(\approx 0.1 \mathrm{~kJ} / \mathrm{g}$-atom) because the specific heat capacity difference between crystal and melt is small at the melting point.

Figure 4 shows the calculated entropy of the undercooled $\mathrm{Zr}_{41.2} \mathrm{Ti}_{13.8} \mathrm{Cu}_{12.5} \mathrm{Ni}_{10.0} \mathrm{Be}_{22.5}$ melt with respect to the crystal. The entropy of the undercooled liquid decreases with increasing undercooling until it reaches the entropy of the crystal at the Kauzmann temperature, $T_{K}$.

The existence of the undercooled liquid below this temperature would violate the Kauzmann paradox ${ }^{6}$ suggesting $T_{K}$ to be the lower bound for the glass transition for thermodynamic reasons. This is due to the fact that the liquid should not have a smaller entropy than the crystal. An alloy, which could be kept as an undercooled liquid from $T_{\dot{m}}$ down to $T_{K}$, has to undergo the transition into the amorphous state by a sudden drop of the specific heat capacity to the value of the glass. For our glass, we obtain a Kauzmann temperature of $560 \mathrm{~K}$, which is below the kinetically observed glass transition temperatures.

The enthalpy difference between the undercooled liquid and the crystal, which decreases with increasing undercooling, is plotted in Fig. 5. The specific heat capacity is integrated from $T_{0}$ down to the Kauzmann temperature. Since the difference in specific heat capacity between amorphous phase and crystal is only $1 \mathrm{~J} / \mathrm{g}$-atom. $\mathrm{K}$ the enthalpy difference remains virtually constant for temperatures below $T_{K}$. However, the extrapolated enthalpy below about $620 \mathrm{~K}$ can hardly be achieved in the real experiment. The curye below $620 \mathrm{~K}$ belongs to an ideal undercooled liquid and glass, respectively, that requires extremely slow cooling rate to form (see next paragraph). In the real experiment, the liquid freezes to a glass throughout the glass transition, and the larger the cooling rate, the more residual enthalpy and entropy are frozen in. In Fig. 5, the measured heats of crystallization are included (triangles), indicating a good agreement

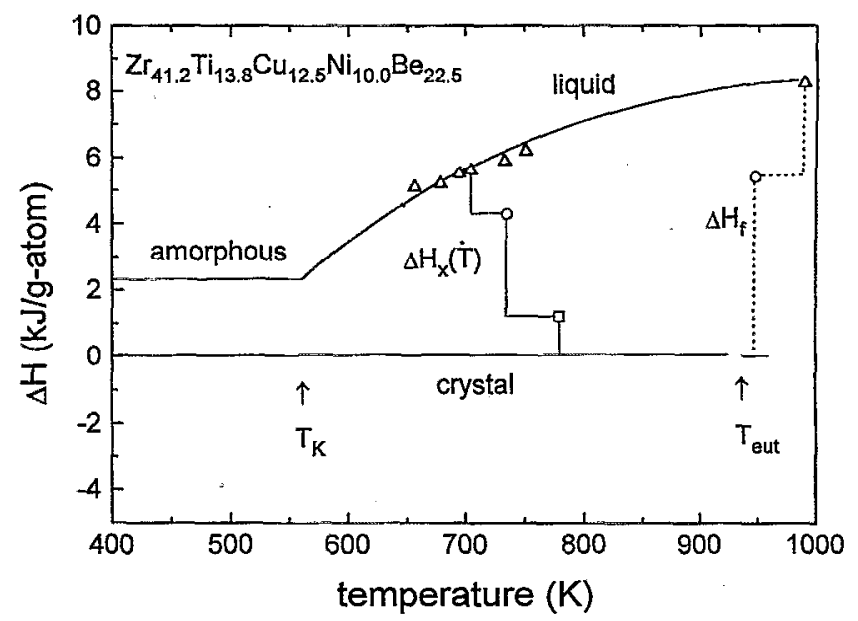

FIG. 5. Enthalpy of the undercooled liquid with respect to the crystal, including the overall heats of crystallization depending on heating rates $(\Delta)$. The paths of crystallization and melting are marked according to the different transformation steps for a rate of $0.33 \mathrm{~K} / \mathrm{s}$.

with the independently obtained $\Delta H$ curve. With increasing heating rates, the samples start to crystallize at higher temperatures from the undercooled liquid, which is already in metastable equilibrium. This explains the observed heating rate dependence of the overall heat of crystallization.

The calculated Gibbs free energy function with respect to the crystalline state is plotted in Fig. 6. Since there is a finite and increasing difference in specific heat capacity between the melt and the crystal on undercooling, the Turnbull approximation $\left(\Delta c_{p}^{i-x}=0 \text {, for } T \leqslant T_{m}\right)^{11}$ is only valid for undercoolings of about $100 \mathrm{~K}$. For larger undercoolings, the real Gibbs free energy difference becomes smaller due to the relative stabilization of the undercooled melt. This stabilization is caused by the increasing specific heat capacity that is attributed to a decreasing free volume, and most likely a gradual gain of short range order in the alloy melt. The observed Gibbs free energy difference is, for example, $1.5 \mathrm{~kJ} /$ g-atom at $0.8 T_{m}$. This value is relatively small compared to

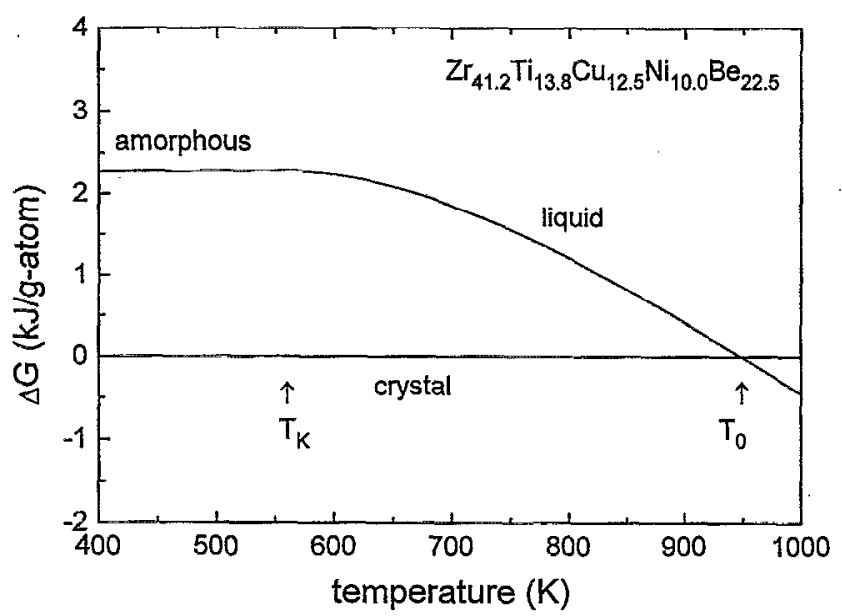

FIG. 6. Gibbs free energy of the undercooled liquid with respect to the crystal as a function of temperature. 


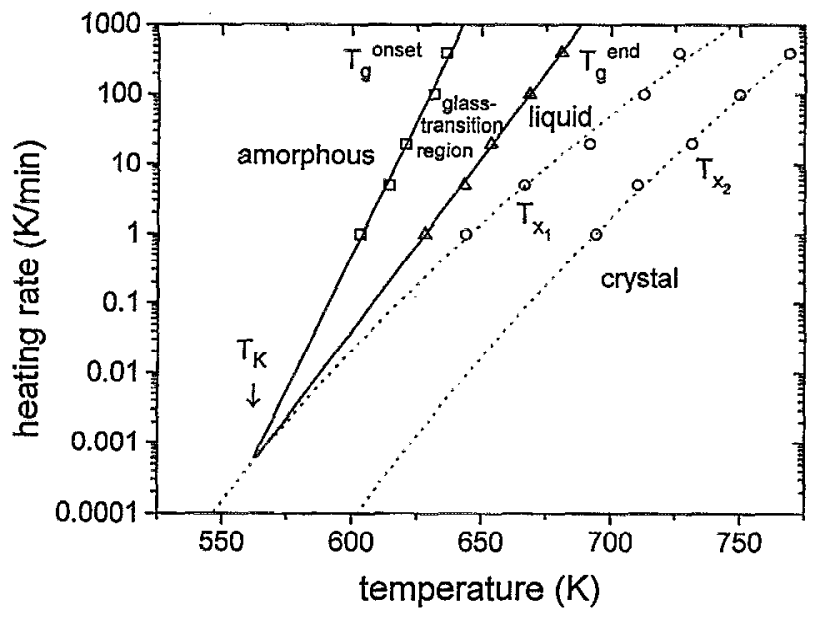

FIG. 7. Kinetical map of the glass transition and the first two crystallization peaks. Onset and end temperatures of the glass transition are fitted linearly to the logarithm of the heating rate. The heating rate dependence of the crystallization is fitted according to an Arrhenius law.

conventional binary glass forming alloys like $\mathrm{Ni}_{50} \mathrm{Ti}_{50}$ or $\mathrm{Nb}_{50} \mathrm{Ni}_{50}$ at $0.8 T_{m}$, where Gibbs free energy differences of $2.5 \mathrm{~kJ} / \mathrm{g}$-atom ${ }^{12}$ and $3.2 \mathrm{~kJ} / \mathrm{g}$-atom, ${ }^{10}$ respectively, are found. The small Gibbs free energy difference turns out to be one crucial point in understanding the high glass forming ability in BMG formers, and will be the subject of further investigations.

\section{B. Glass transition and Kauzmann temperature}

In the following, the calculated Kauzmann temperature is compared with the kinetically observed glass transition. Onset and end temperatures of the glass transition, as well as crystallization, as measured in the DSC experiments for heating rates between 0.0167 and $6.667 \mathrm{~K} / \mathrm{s}$ are plotted in Fig. 7 . We find that both $T_{g}^{\text {onset }}$ and $T_{g}^{\text {end }}$ depend linearly on the logarithm of the heating rate of the experiment. This behavior of the glass transition temperature was also found by other authors for metallic and nonmetallic systems as well ${ }^{13,14}$ and is discussed by Jäckle. ${ }^{15}$

The slopes of the curves describing the heating rate dependence of $T_{g}^{\text {onset }}$ and $T_{g}^{\text {end }}$ in Fig. 7 are different, which is due to the fact that the width of the glass transition region becomes smaller with decreasing heating rate. 'The extrapolation of both curves to lower temperatures and heating rates leads to a point of intersection. Here, the width of the glass transition region becomes zero, suggesting that the specific heat capacity would step from the value of the glass to the undercooled liquid. The temperature that corresponds to the point of intersection is $562 \mathrm{~K}$. This is about the value of the Kauzmann temperature within the experimental error. The heating rate to reach this lower limit for the glass transition temperature would be $1.67 \times 10^{-5} \mathrm{~K} / \mathrm{s}$. It would be extremely difficult to observe this ideal glass transition experimentally, although it might be theoretically accessible since the extrapolated onset temperature of the primary crystallization also intersects both $T_{g}^{\text {onset }}$ and $T_{g}^{\text {end }}$ close to their point of intersection (see Fig. 7).

\section{SUMMARY AND CONCLUSIONS}

The specific heat capacities of the undercooled liquid, the amorphous state and the crystal of the $\mathrm{Zr}_{41.2} \mathrm{Ti}_{13.8} \mathrm{Cu}_{12.5} \mathrm{Ni}_{10.0} \mathrm{Be}_{22.5}$ bulk metallic glass former were measured by differential scanning calorimetry. In addition, the glass transition temperatures, crystallization temperatures, heats of crystallization, and heats of fusion were measured as a function of heating rate. Based on the thermodynamic data the thermodynamic functions of the undercooled liquid were calculated using a $1 / T^{2}$ dependence of the specific heat capacity in the undercooled liquid. The calculations show that the Gibbs free energy difference between liquid and solid state stays small even for large undercoolings. For example, $\Delta G$ is $1.5 \mathrm{~kJ} / \mathrm{g}$-atom at $0.8 T_{m}$. This relatively small Gibbs free energy difference appears to be a contrib. uting factor in the high glass forming ability of the alloy. The Kauzmann temperature of the $\mathrm{Zr}_{41.2} \mathrm{Ti}_{13.8} \mathrm{Cu}_{12.5} \mathrm{Ni}_{10.0} \mathrm{Be}_{22.5}$ alloy is calculated to be $560 \mathrm{~K}$, representing the lower bound for the kinetically observed glass transition. This is deduced from extrapolating the rate dependence of onset and end temperature to low heating rates. Both onset and end temperatures depend linearly on the logarithm of heating rate and intersect at the Kauzmann temperature.

\section{ACKNOWLEDGMENTS}

The authors would like to thank D. Isheim and S. Friedrichs for their assistance on the DSC experiments and U. Geyer, S. Schneider, E. Bakke, and H. Fecht for valuable help and fruitful discussions. This work was supported by the German Alexander von Humboldt Foundation via the Feodor Lynen Program, the Department of Energy (Grant No. DEFG-03-86ER45242) and the National Aeronautics and Space Administration (Grant No. NAG8-954).

'A. Inoue, T. Zhang, and T. Masumoto, Mater. Trans. JM 31, 425 (1991).

${ }^{2}$ T. Zhang, A. Inoue, and T. Masumoto, Mater. Trans. JIM 32, 1005 (1991).

${ }^{3}$ A. Peker and W. L. Johnson, Appl. Phys. Lett. 63, 2342 (1993).

${ }^{4}$ Y. J. Kim, R. Busch, W. L. Johnson, A. J. Rulison, and W. K. Rhim, Appl. Phys. Lett. 65, 2136 (1994).

${ }^{5}$ W. L. Johnson and A. Peker, NATO Workshop on Science and Technology of Rapid Solidification and Processing, West Point, June 21-24, 1994.

${ }^{6}$ W. Kauzmann, Chem. Rev. 43, 219 (1948).

${ }^{7}$ H. Fecht (private communication).

${ }^{2} \mathrm{O}$. Kubaschewski, C. B. Alcock, and P. J. Spencer, Materials Thermochemistry, 6th ed. (Pergamon, New York, 1993).

${ }^{9}$ R. Bormann, F. Gärtner, and K. Zöltzer, J. Less-Common Metals 145, 19 (1988).

${ }^{10}$ R. Busch and R. Bormann (unpublished research).

${ }^{11}$ D. Turnbull, J. Appl. Phys. 21, 1022 (1950).

${ }^{12}$ R. Bormann and K. Zöltzer, Phys. Status Solidi A 131, 691 (1992).

${ }^{13}$ G. S. Grest and M. H. Cohen, Phys. Rev. B 21, 4113 (1980).

${ }^{14}$ R. Lück, Q. Jiang, and B. Predel, J. Non-Cryst. Solids 117/118, 911 (1990).

${ }^{15}$ J. Jäckle, Rep. Prog. Phys. 49, 171 (1986). 